\title{
Enhancement of images using various histogram equalization techniques
}

\author{
Deepak Kumar Behera \\ ( Department of Electronics and Communication Engineering ,SBSSTC, Ferozepur, Punjab, India)
}

\begin{abstract}
Image enhancement is a mean as the improvement of an image appearance by increasing dominance of some features or by decreasing ambiguity between different regions of the image. Image enhancement processes consist of a collection of techniques that seek to improve the visual appearance of an image or to convert the image to a form better suited for analysis by a human or machine. Various enhancement schemes are used for enhancing an image which includes gray scale manipulation, filtering and Histogram Equalization (HE). Histogram equalization is one of the well known image enhancement technique. It became a popular technique for contrast enhancement because this method is simple and effective. This paper presents a review of histogram equalization techniques for image enhancement.
\end{abstract}

Keywords: image enhancement, contrast enhancement, histogram equalization,

\section{Introduction}

Histogram is one of the important features which are very related to image enhancement. The histogram does not only gives us a general overview on some useful image statistics (e.g. mode, and dynamic range of an image), but it also can be used to predict the appearance and intensity characteristic of an image. If the histogram is concentrated on the low side of the intensity scale, the image is mostly a dark image. On the other hand, if the histogram is concentrated on the high side of the scale, the image is mostly a bright image. If the histogram has a narrow dynamic range, the image usually is an image with a poor contrast [1].

In order to define a histogram, first, assume that $X=\{X(i, j)\}$ is an image that is composed of $L$ discrete gray levels denoted by $\left\{X_{0}, X_{1}, \ldots, X_{L-1}\right\}$. Here, $X(i, j)$ represents the intensity of the image at spatial location $(i, j)$ with the condition that $X(i, j) \in\left\{X_{0}, X_{1}, \ldots, X_{L-1}\right\}$. As the intensities are all in discrete values, the histogram of a digital image is a discrete function. Then, the histogram is $\mathrm{h}$ defined as:

$$
\mathrm{h}\left(\mathrm{X}_{k}\right)=\mathrm{n}_{k} \text {, for } \mathrm{k}=0,1, \ldots \ldots, \mathrm{L}-1
$$

where $\mathrm{X}_{k}$ is the $k$-th gray level and $\mathrm{n}_{k}$ presents the number of times that the gray level $\mathrm{X}_{k}$ appears in the image. In other words, the histogram is the frequency of occurrence of the gray levels in the image [2].Alternatively, as used by Wang et al. [3], and Wang and Ye [4], the histogram also can be defined as the statistic probability distribution of each gray level in a digital image. For a given image $\mathbf{X}$, the Probability Density Function (PDF) for intensity $\mathrm{X}_{k}, \mathrm{p}\left(\mathrm{X}_{k}\right)$, is given by

$$
\mathrm{p}\left(\mathrm{X}_{k}\right)=\mathrm{n}_{k} / \mathrm{N} \text {,for } \mathrm{k}=0,1, \ldots \ldots, \mathrm{L}-1
$$

where $\mathrm{N}$ is the total number of samples in the image. By comparing equation (2) with equation (1), the PDF is actually a normalized version of the histogram. Usually, the histogram of an image $\mathbf{X}$ is presented as a graph plots of $\mathrm{h}\left(\mathrm{X}_{k}\right)$ versus $\mathrm{X}_{k}$, or $\mathrm{p}\left(\mathrm{X}_{k}\right)$ versus $\mathrm{X}_{k}$.

\section{Histogram Equalization(HE)}

Histogram Equalization is a technique that generates a gray map which changes the histogram of an image and redistributing all pixels values to be as close as possible to a user-specified desired histogram. HE allows for areas of lower local contrast to gain a higher contrast. Histogram equalization automatically determines a transformation function seeking to produce an output image with a uniform Histogram. Histogram equalization is a method in image processing of contrast adjustment using the image histogram. This method usually increases the global contrast of many images, especially when the usable data of the image is represented by close contrast values. Through this adjustment, the intensities can be better distributed on the histogram. Histogram equalization accomplishes this by effectively spreading out the most frequent intensity values. Histogram equalization automatically determines a transformation function seeking to produce an output image with a uniform Histogram. 


\section{Histogram equalization techniques}

There are numerous methods by which Histogram of an image can be equalized. Depending upon the area of Application, we can choose the different histogram equalization techniques. We will see the following types of Histogram Equalization methods in details.

A. Classical Histogram Equalization(CHE): CHE is the fundamental technique for image processing, especially when gray level images are considered. The main purpose of this method is to distribute the given number of gray levels over a range uniformly, thus enhancing its contrast. The CHE tries to produce an output image with a flattened histogram, means a uniform distribution. An image is formed by the dynamic range of values of gray levels. Basically, the entire gray levels are denoted as 0 to $L-1$.

B. Adaptive Histogram Equalization(AHE): Adaptive Histogram Equalization (AHE) is used to improve contrast in images. It computes many ordinary histograms, each one analogous with a section of the image. Thus, the output results in each to redistributing the lightness values. It is appropriate to adjust the local contrast and to fetch clear details. The contrast, especially in homogeneous areas, can be limited in order to avoid amplifying the noise which might be present in the image. On the other hand, AHE is responsible for overamplifying noise in some homogeneous regions of an image.

C. Bi-Histogram Equalization(BHE): This is done by dividing the mean gray level and then applying CHE method on each of the two sub-sectioned image. Its objective is to produce method suitable for real-time applications. But again this method has the same disadvantage as CHE by inputting unwanted signals. The major basis of origination of this method is to overcome the drawback introduced by CHE. Here, the original image is segmented twice i.e into two sub-sections.

D. Brightness Preserving Bi-Histogram Equalization (BBHE): This method divides the image histogram into two parts. Here, the separation intensity is presented by the input mean brightness value, which is the average intensity of all pixels that construct the input image[5].After this separation process, these two histograms are independently equalized. By doing this, the mean brightness of the resultant image will lie between the input mean and the middle gray level. The histogram with range from 0 to L-1 is divided into two parts, with separating intensity. This separation produces two histograms. The first histogram has the range of 0 to, while the second histogram has the range of to L-1.

\section{E. Minimum Mean Brightness Error Bi-Histogram Equalization(MMBEBHE):}

Minimum Mean Brightness Error Bi-Histogram Equalization (MMBEBHE)algorithm was proposed to overcome the limitation of BBHE method .Becase there are still cases that are not handled well by BBHE.The MMBEBHE provide maximum brightness preservation. BBHE method separates the input image's histogram into two based on input mean before equalizing them independently. However, using input mean as the threshold level to separate the histogram does not guarantee to give maximum brightness preservation. Here described brightness preservation is based on an objective measurement referred as Absolute Mean Brightness Error (AMBE). It is defined as the absolute difference between the input and the output mean as follow:

$\mathrm{AMBE}=(\mathrm{X}, \mathrm{Y})=\mathrm{Mx}-\mathrm{My}$

Where Mx is the mean of input and My is the mean of output image.

Lower AMBE implies the better brightness preservation.

F. Dualistic sub-image histogram equalization(DSIHE): This is a novel histogram equalization technique in which the original image is decomposed into two equal area sub-images based on its gray level probability density function. Then the two sub-images are equalized respectively. At last, we get the result after the processed sub-images are composed into one image. In fact, the algorithm can not only enhance the image visual information effectively, but also constrain the original image's average luminance from great shift. This makes it possible to be utilized in video system directly.

G. Recursive Mean Separate Histogram Equalization(RMSHE): This is an extended version of the BHE method proposed before, and named as recursive mean-separate HE (RMSHE), Instead of decomposing the image only once, the RMSHE method offers to perform image decomposition recursively, up to a scale $r$, generating $2 r$ sub images. After, each one of these sub-images is independently enhanced using the CHE method. Note that, computationally speaking, this method presents a problem: the number of decomposed subhistograms is a power of two.

H. Multi-Decomposition Histogram Equalization(MDHE): All the HE methods that we have covered prior to this, enhances the contrast of an image but are unable to preserve its brightness. As a result, these methods can generate unnatural and non existing objects in the processed image. To eliminate these limitations, MDHE comes up with a novel technique by decomposing the image into various small images. Then the image contrast enhancement provided by CHE in each sub-image is less concentrated, leading the output image to have a more likely and acceptable look. In this method 4 step process is carried out as given below sequentially from 1 to 4

(1) Multi-Decomposition of the image

(2)Applying histogram based techniques

(3)Interpolating the image 
(4)Brightness Preservation

I. Dynamic Histogram Equalization(DHE): The Dynamic Histogram Equalization (DHE) technique takes control over the effect of traditional Histogram equalization so that it performs the enhancement of an image without making any loss of details in it. It employs a partitioning operation over the input histogram to chop it into some sub histograms so that they have no dominating component in them. Then each sub histogram is allotted a dynamic gray level (GL) which further can be mapped by Histogram equalization [8]. This is done by distributing total available dynamic range of gray levels among the sub-histograms based on their dynamic range in input image and cumulative distribution (CDF) of histogram values. This allotment of stretching range of contrast prevents small features of the input image from being dominated and washed out, and ensures a moderate contrast enhancement of each portion of the whole image. At last, for each sub-histogram a separate transformation function is calculated based on the traditional HE method and gray levels of input image are mapped to the output image accordingly. The whole technique can be divided in three parts partitioning the histogram, allocating GL ranges for each sub histogram and applying Histogram equalization on each of them. A better overall contrast enhancement is gained by DHE with controlled dynamic range of gray levels and eliminating the possibility of the low histogram components being compressed that may cause some part of the image to have washed out appearance.

J. Brightness Preserving Dynamic Histogram Equalization(BPDHE): The brightness preserving dynamic histogram equalization (BPDHE) is an extension to HE, which fulfils the requirement of maintaining the mean brightness of the image, by producing the output image with the mean intensity almost equal to the mean intensity of the input. BPDHE is specificaly an extension to both MPHEBP and DHE. Similar to MPHEBP, this method partitions the histogram based on the local maximums of the smoothed histogram. However, before the histogram equalization taking place, the method will map each partition to a new dynamic range, similar to DHE. Since the change in the dynamic range will cause the change in mean brightness, the final step of this method involves the normalization of the output intensity. So, the average intensity of the resultant image will be same as the input. With this criterion, BPDHE will produce better enhancement and better in preserving the mean brightness compared with DHE.

K. Mean brightness preserving histogram equalization (MBPHE): MBPHE is a novel extension to HE. This type of enhancement method is specially developed for the use in consumer electronic products such as digital television, digital camera and camcorder. The mean brightness preserving histogram equalization (MBPHE) methods basically can be divided into two main groups, which are bisections MBPHE, and multi-sections MBPHE. Bisections MBPHE group is the simplest group of MBPHE [6]. Basically, these methods separate the input histogram into two sections. These two histogram sections are then equalized independently. However, bisections MBPHE can preserve the mean brightness only to a certain extent. However, some cases do require higher degree of preservation to avoid unpleasant artifacts. Furthermore, bisections MBPHE can only preserve the original mean brightness if and only if the input histogram has a quasi-symmetrical distribution around its separating point. But, most of the input histograms do not have this property. This condition leads to the failure of bisections MBPHE in preserving the mean intensity in real life applications. Multi-sections MBPHE group has a better mean brightness preservation as compared with the group of bisections MBPHE. In multi-sections MBPHE, the input histogram is divided into $\mathrm{R}$ sub-histograms, where $\mathrm{R}$ is any positive integer value. Then each sub-histogram is equalized independently. The creation of the sub -histograms can be carried out recursively (e.g. by using the mean or median intensity value), or based on the shape of the input histogram itself (e.g. using the locations of local maximum or local minimum). Yet, in these methods, the detection of the separating points process normally requires complicated algorithms, which then associated with relatively high computational time. Furthermore, these methods usually increase the hardware requirement in the implementations for consumer electronic products.

\section{Conclusions and Future Applications}

In this Paper, a frame work for image enhancement based on prior knowledge on the Histogram Equalization has been presented .Histogram Equalization works on the various elements of images: saturation, contrast, sharpness and brightness. Above HE methods focus on these four parameters and thus, enhance the quality of images. The desired contrast levels, along with preservation of brightness and not only this, the natural look of the input image can be maintained by using the above histogram equalization techniques. Future applications include photos obtained from satellite communications that are distorted due to space interference and dispersion losses can be regenerated with their natural look. Other application fields are Discoveries of Geo-stationary bodies, Medical field- X-Rays, weather information, surveillance camera processing. 


\section{References:}

[1] R. C. Gonzalez and R. E. Woods, Digital image processing, 2nd ed. Boston, MA, USA: Prentice-Hall of India, 2002.

[2] David Menotti, Laurent Najman, Jacques Facon, and Araujo de A. Araujo, "Multi histogram equalization methods for contrast enhancement and brightness preserving”, IEEE Trans. Consumer Electronics, vol. 53, no. 3, pp. 1186 - 1194, August 2007.

[3] Y. Wang, Q. Chen, and B. Zhang, "Image enhancement based on equal area dualistic sub-image histogram equalization method," Consumer Electronics, IEEE Transactions on, vol. 45, no. 1, pp. 68-75,1999.

[4] Wang and Z. Ye, "Brightness preserving histogram equalization with maximum entropy: a variational perspective," Consumer Electronics, IEEE Transactions on, vol. 51, no. 4, pp. 1326-1334, 2005.

[5] Yeong -Taeg Kim , “Contrast Enhancement using Brightness Preserving Bi- Histogram equalization”, IEEE trans. on consumer Electronics, Vol. 43 ,pp.1-8 1997

[6] Manpreet Kaur, Jasdeep Kaur, Jappreet Kaur , "Survey of Contrast Enhancement Techniques based on Histogram Equalization" (IJACSA), 2011, Vol. 2 No. 7,pp 136

[7] S.-D. Chen and A. Ramli, "Minimum mean brightness error Bi-Histogram equalization in contrast enhancement," IEEE Trans. on Consumer Electronics, vol. 49, no. 4, pp. 1310-1319, Nov. 2003

[8] M. Abdullah-Al-Wadud, Md. Hasanul Kabir, M. Ali Akber Dewan, and Oksam Chae, "A dynamic histogram equalization for image contrast enhancement”, IEEE Trans. Consumer Electron., vol. 53, no. 2, pp. 593- 600, May 2007.

[9]. J.-Y. Kim, L.-S. Kim, and S.-H. Hwang, "An advanced contrast enhancement using partially overlapped sub-block histogram equalization,” IEEE Trans. Circuits Syst. Video Technol., vol. 11, no. 4,pp. 475-484, Apr. 2001.

[10]. S.-D. Chen and A. Ramli, "Contrast enhancement using recursive Mean-Separate histogram equalization for scalable brightness preservation,"IEEE Trans. on Consumer Electronics, vol. 49, no. 4, pp. 1301-1309,Nov. 2003.

[11]. Chao Wang and Zhongfu Ye, "Brightness preserving histogram equalization with maximum entropy: a variational perspective", IEEE Trans. Consumer Electronics, vol. 51, no. 4, pp. 1326-1334, November2005.

[12]. K. Wongsritong, K. Kittayaruasiriwat, F. Cheevasuvit, K. Dejhan, and A.Somboonkaew, "Contrast enhancement using multi peak histogram equalization with brightness preserving", The 1998 IEEE Asia-Pasific Conference on Circuit and Systems, pp. 24-27, November 1998 .

[13] K. S. Sim, C. P. Tso, and Y. Y. Tan. "Recursive sub-image histogram equalization applied to gray scale images", Pattern Recognition Letters, vol. 28, no. 10, pp. 1209-1221, July 2007..

[14] Chun-Ming Tsai, and Zong-Mu Yeh, "Contrast enhancement by automatic and parameter-free piecewise linear transformation for color images", IEEE Trans. Consumer Electronics, vol. 54, no. 2, pp. 213-219, May 2008.

[15]. Haidi Ibrahim, and Nicholas Sia Pik Kong, "Brightness preserving dynamic histogram equalization for image contrast enhancement”, IEEE Trans. Consumer Electronics, vol. 53, no. 4, pp. 1752 - 1758, November 2007.

[16] Nicholas Sia Pik Kong, and Haidi Ibrahim, "Improving the visual quality of abdominal magnetic resonance images using histogram equalization", In Technology and Applications in Biomedicine, 2008. ITAB 2008.International Conference on, pp. 138-139, Shenzhen, China, May 2008.

[17] Nicholas Sia Pik Kong, and Haidi Ibrahim, "Color image enhancement using brightness preserving dynamic histogram equalization", IEEE Trans. Consumer Electronics, vol. 54, no. 4, pp. 1962 - 1968, November 2008.

[18]. Nymlkhagva Sengee, and Heung Kook Choi, "Brightness preserving weight clustering histogram equalization", IEEE Trans. Consumer Electronics, vol. 54, no. 3, pp. 1329 - 1337, August 2008.

[19]. Gyu-Hee Park, Hwa-Hyun Cho, and Myung-Ryul Choi, "A contrast enhancement method using dynamic range separate histogram equalization”, IEEE Trans. Consumer Electronics, vol. 54, no. 4, pp. 1981 - 1987, November 2008.

[20]. Chen Hee Ooi, Nicholas Sia Pik Kong, and Haidi Ibrahim, "Bi-Histogram Equalization with a Plateau Limit for Digital Image Enhancement”, IEEE Trans. Consumer Electronics, Vol. 55, No. 4, NOVEMBER 2009.

[21]. Vossepoel,Albert M.Stoel,B.C.;Meershoek,A.P."Adaptive histogram equalization using variable regions", IEEE conference in1988.

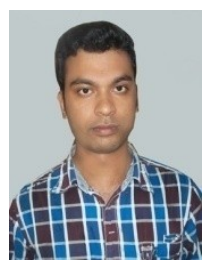

Er Deepak Kumar Behera received his Bachelor's degree( B.Tech) from Biju Patnaik University of Technology,Raurkela,Odisha(India)with specialization in Electronics and Telecommunication engineering in year 2010,and Currently he is pursuing M.Tech from Saheed Bhagat Singh State Technical Campus (formerly, SBS College of Engineering \& Technology) Ferozepur, Punjab (India) with specialization in Electronics \& Communication Engineering. His fields of special interest include Image Processing,Video processing, Artificial neural network, fuzzy logic, Evolutionary Algorithm, Genetic algorithm. 